\title{
Delay-Induced Oscillations in a Competitor-Competitor-Mutualist Lotka-Volterra Model
}

\author{
Changjin Xu \\ Guizhou Key Laboratory of Economics System Simulation, Guizhou University of Finance and Economics, Guiyang 550004, China \\ Correspondence should be addressed to Changjin Xu; xcj403@126.com
}

Received 14 January 2017; Accepted 30 March 2017; Published 26 April 2017

Academic Editor: Alicia Cordero

Copyright (c) 2017 Changjin Xu. This is an open access article distributed under the Creative Commons Attribution License, which permits unrestricted use, distribution, and reproduction in any medium, provided the original work is properly cited.

\begin{abstract}
This paper deals with a competitor-competitor-mutualist Lotka-Volterra model. A series of sufficient criteria guaranteeing the stability and the occurrence of Hopf bifurcation for the model are obtained. Several concrete formulae determine the properties of bifurcating periodic solutions by applying the normal form theory and the center manifold principle. Computer simulations are given to support the theoretical predictions. At last, biological meaning and a conclusion are presented.
\end{abstract}

\section{Introduction}

The study of dynamical behaviors for tremendous predatorprey models has been a hot issue in population dynamics in the past few decades. Many results have been reported [1-11]. In the real world, any biological or environmental parameters are naturally subject to fluctuation in time. The effects of a periodically varying environment are important for evolutionary theory as the selective forces on systems in a fluctuating environment differ from those in a stable environment. Meanwhile, time delay due to gestation is common example because, generally, the consumption of prey by the predator throughout its past history governs the present birth rate of the predator. Based on all the above point, $\mathrm{Lv}$ et al. [12] had investigated the periodic solution of the following competitor-competitor-mutualist Lotka-Volterra model by using Krasnoselskii's fixed point theorem

$$
\begin{aligned}
& \dot{x}_{1}(t)=x_{1}(t)\left[r_{1}(t)-a_{11}(t) x_{1}\left(t-\tau_{11}(t)\right)\right. \\
& \left.\quad-a_{12}(t) x_{2}\left(t-\tau_{12}(t)\right)+a_{13}(t) x_{3}\left(t-\tau_{13}(t)\right)\right], \\
& \dot{x}_{2}(t)=x_{2}(t)\left[r_{2}(t)-a_{21}(t) x_{1}\left(t-\tau_{21}(t)\right)\right. \\
& \left.\quad-a_{22}(t) x_{2}\left(t-\tau_{22}(t)\right)+a_{23}(t) x_{3}\left(t-\tau_{23}(t)\right)\right], \\
& \dot{x}_{3}(t)=x_{3}(t)\left[r_{3}(t)+a_{31}(t) x_{1}\left(t-\tau_{31}(t)\right)\right. \\
& \left.\quad+a_{32}(t) x_{2}\left(t-\tau_{32}(t)\right)-a_{33}(t) x_{3}\left(t-\tau_{33}(t)\right)\right],
\end{aligned}
$$

where $x_{1}(t)$ and $x_{2}(t)$ denote the densities of competing species at time $t$ and $x_{3}(t)$ denotes the density of cooperating species at time t. $r_{i}, a_{i j} \in C(R,[0, \infty))$ and $\tau_{i j} \in C(R, R)$ are $\omega$-periodic functions $(\omega>0)$. The parameters $\tau_{i j}(t) \geq 0(i=$ $1,2,3 ; j=1,2,3)$ are the feedback time delay of different species. In detail, one can see [12].

It is well known that the research on the Hopf bifurcation, especially on the stability of bifurcating periodic solutions and direction of Hopf bifurcation, is one of the most important themes on the predator-prey dynamics. There are a great deal of papers which deal with this topic [11, 13-22]. The purpose of this paper is to discuss the stability and the properties of Hopf bifurcation of model (1). To simplify the analysis for model (1), we make the following assumptions: all biological and environmental parameters are constants in time and only the feedback time delay of competing species $x_{i}(i=1,2)$ to the growth of the species itself and the feedback time delay of cooperating species $x_{3}$ to the growth of the species itself exist and are the same. Then system (1) can be described as the form

$$
\begin{aligned}
& \dot{x}_{1}(t) \\
& \quad=x_{1}(t)\left[r_{1}-a_{11} x_{1}(t-\tau)-a_{12} x_{2}(t)+a_{13} x_{3}(t)\right], \\
& \dot{x}_{2}(t) \\
& \quad=x_{2}(t)\left[r_{2}-a_{21} x_{1}(t)-a_{22} x_{2}(t-\tau)+a_{23} x_{3}(t)\right], \\
& \dot{x}_{3}(t) \\
& \quad=x_{3}(t)\left[r_{3}+a_{31} x_{1}(t)+a_{32} x_{2}(t)-a_{33} x_{3}(t-\tau)\right] .
\end{aligned}
$$


In this paper, we consider the effect of time delay $\tau$ on the dynamics of system (2). We not only give the conditions on the stability of the positive equilibrium of (2) and the existence of periodic solutions but also derive the formulae for determining the properties of a Hopf bifurcation.

The remainder of the paper is organized as follows. In Section 2, we investigate the stability of the positive equilibrium and the occurrence of local Hopf bifurcations. In Section 3, the direction and stability of the local Hopf bifurcation are established. In Section 4, numerical simulations are carried out to illustrate the validity of the main results. Biological explanations and some main conclusions are drawn in Section 5.

\section{Stability of the Positive Equilibrium and Local Hopf Bifurcations}

Consider the realistic implication and actual application of biological system; in this section, we shall only study the stability of the positive equilibrium and the existence of local Hopf bifurcations. It is easy to see that system (2) has a unique positive equilibrium $E_{0}\left(x_{1}^{*}, x_{2}^{*}, x_{3}^{*}\right)$ if the condition

$$
\operatorname{sign}\{\Delta\}=\operatorname{sign}\left\{\Delta_{1}\right\}=\operatorname{sign}\left\{\Delta_{2}\right\}=\operatorname{sign}\left\{\Delta_{3}\right\}
$$

holds, where

$$
\begin{gathered}
\Delta=\operatorname{det}\left(\begin{array}{lll}
a_{11} & a_{12} & -a_{13} \\
a_{21} & a_{22} & -a_{23} \\
a_{31} & a_{31} & -a_{33}
\end{array}\right), \\
\Delta_{1}=\operatorname{det}\left(\begin{array}{lll}
r_{1} & a_{12} & -a_{13} \\
r_{2} & a_{22} & -a_{23} \\
-r_{3} & a_{31} & -a_{33}
\end{array}\right), \\
\Delta_{2}=\operatorname{det}\left(\begin{array}{lll}
a_{11} & r_{1} & -a_{13} \\
a_{21} & r_{2} & -a_{23} \\
a_{31} & -r_{3} & -a_{33}
\end{array}\right), \\
\Delta_{3}=\operatorname{det}\left(\begin{array}{lll}
a_{11} & a_{12} & r_{1} \\
a_{21} & a_{22} & r_{2} \\
a_{31} & a_{31} & -r_{3}
\end{array}\right) .
\end{gathered}
$$

Let $\bar{x}_{1}(t)=x_{1}(t)-x_{1}^{*}, \bar{x}_{2}(t)=x_{2}(t)-x_{2}^{*}$, and $\bar{x}_{3}(t)=x_{3}(t)-$ $x_{3}^{*}$ and still denote $\bar{x}_{i}(t)(i=1,2,3)$ by $x_{i}(t)(i=1,2,3)$, and then (2) takes the form

$$
\begin{aligned}
\dot{x}_{1}(t)= & b_{1} x_{1}(t-\tau)+b_{2} x_{2}(t)+b_{3} x_{3}(t) \\
& +b_{4} x_{1}(t) x_{1}(t-\tau)+b_{5} x_{1}(t) x_{2}(t) \\
& +b_{6} x_{1}(t) x_{3}(t), \\
\dot{x}_{2}(t)= & c_{1} x_{1}(t)+c_{2} x_{2}(t-\tau)+c_{3} x_{3}+c_{4} x_{1}(t) x_{2}(t) \\
& +c_{5} x_{2}(t) x_{2}(t-\tau)+c_{6} x_{2}(t) x_{3}(t),
\end{aligned}
$$

$$
\begin{aligned}
\dot{x}_{3}(t)= & d_{1} x_{1}(t)+d_{2} x_{2}(t)+d_{3} x_{3}(t-\tau) \\
& +d_{4} x_{1}(t) x_{3}(t)+d_{5} x_{2}(t) x_{3}(t) \\
& +d_{6} x_{3}(t) x_{3}(t-\tau),
\end{aligned}
$$

where

$$
\begin{aligned}
& b_{1}=-a_{11} x_{1}^{*}, \\
& b_{2}=-a_{12} x_{1}^{*}, \\
& b_{3}=a_{13} x_{1}^{*}, \\
& b_{4}=-a_{11}, \\
& b_{5}=-a_{12}, \\
& b_{6}=a_{13}, \\
& c_{1}=-a_{21} x_{2}^{*}, \\
& c_{2}=-a_{22} x_{2}^{*}, \\
& c_{3}=a_{23} x_{2}^{*}, \\
& c_{4}=-a_{21}, \\
& c_{5}=-a_{22}, \\
& c_{6}=a_{23}, \\
& d_{1}=a_{31} x_{3}^{*}, \\
& d_{2}=a_{32} x_{3}^{*}, \\
& d_{3}=-a_{33} x_{3}^{*}, \\
& d_{4}=a_{31}, \\
& d_{5}=a_{32}, \\
& d_{6}=-a_{33},
\end{aligned}
$$

The linearization of $(6)$ near $(0,0,0)$ is given by

$$
\begin{aligned}
& \dot{x}_{1}(t)=b_{1} x_{1}(t-\tau)+b_{2} x_{2}(t)+b_{3} x_{3}(t), \\
& \dot{x}_{2}(t)=c_{1} x_{1}(t)+c_{2} x_{2}(t-\tau)+c_{3} x_{3}, \\
& \dot{x}_{3}(t)=d_{1} x_{1}(t)+d_{2} x_{2}(t)+d_{3} x_{3}(t-\tau),
\end{aligned}
$$

whose characteristic equation takes the form

$$
\operatorname{det}\left(\begin{array}{ccc}
\lambda-b_{1} e^{-\lambda \tau} & -b_{2} & -b_{3} \\
-c_{1} & \lambda-c_{2} e^{-\lambda \tau} & -c_{3} \\
-d_{1} & -d_{2} & \lambda-d_{3} e^{-\lambda \tau}
\end{array}\right)=0 .
$$

That is,

$$
\begin{aligned}
\lambda^{3}+m_{1} \lambda+m_{2} & +\left(m_{3} \lambda^{2}+m_{4}\right) e^{-\lambda \tau}+m_{5} \lambda e^{-2 \lambda \tau} \\
+ & m_{6} e^{-3 \lambda \tau}=0,
\end{aligned}
$$


where

$$
\begin{aligned}
& m_{1}=b_{2} c_{1}+c_{3} d_{2}-b_{3} d_{1}, \\
& m_{2}=-b_{1} c_{3} d_{1}-b_{3} d_{2} c_{1}, \\
& m_{3}=-\left(b_{1}+c_{2}+d_{3}\right), \\
& m_{4}=b_{3} c_{2} d_{1}-b_{2} c_{1} d_{3}-b_{1} d_{2} c_{3}, \\
& m_{5}=\left(b_{2}+c_{2}\right) d_{3}+b_{1} c_{2}, \\
& m_{6}=-b_{1} c_{2} d_{3} .
\end{aligned}
$$

Multiplying $e^{\lambda \tau}$ on both sides of (8), it is easy to obtain

$$
\begin{gathered}
\left(\lambda^{3}+m_{1} \lambda+m_{2}\right) e^{\lambda \tau}+m_{3} \lambda^{2}+m_{4}+m_{5} \lambda e^{-\lambda \tau} \\
+m_{6} e^{-2 \lambda \tau}=0 .
\end{gathered}
$$

We need the following lemma to discuss the stability of the positive equilibrium.

Lemma 1 (see [23]). For the transcendental equation

$$
\begin{aligned}
P( & \left.\lambda, e^{-\lambda \tau_{1}}, \ldots, e^{-\lambda \tau_{m}}\right) \\
= & \lambda^{n}+p_{1}^{(0)} \lambda^{n-1}+\cdots+p_{n-1}^{(0)} \lambda+p_{n}^{(0)} \\
& +\left[p_{1}^{(1)} \lambda^{n-1}+\cdots+p_{n-1}^{(1)} \lambda+p_{n}^{(1)}\right] e^{-\lambda \tau_{1}}+\cdots \\
& +\left[p_{1}^{(m)} \lambda^{n-1}+\cdots+p_{n-1}^{(m)} \lambda+p_{n}^{(m)}\right] e^{-\lambda \tau_{m}}=0,
\end{aligned}
$$

as $\left(\tau_{1}, \tau_{2}, \tau_{3}, \ldots, \tau_{m}\right)$ vary, the sum of orders of the zeros of $P\left(\lambda, e^{-\lambda \tau_{1}}, \ldots, e^{-\lambda \tau_{m}}\right)$ in the open right half complex plane can change, only if a zero appears on or crosses the imaginary axis.

For $\tau=0$, (10) becomes

$$
\lambda^{3}+m_{3} \lambda^{2}+\left(m_{1}+m_{5}\right) \lambda+m_{2}+m_{4}+m_{6}=0 .
$$

Obviously, $m_{3}>0$. By the Routh-Hurwitz criteria, it follows that all eigenvalues of (12) have negative real parts if and only if the condition

$$
\begin{aligned}
& m_{2}+m_{4}+m_{6}>0, \\
& m_{3}\left(m_{1}+m_{5}\right)>m_{2}+m_{4}+m_{6}
\end{aligned}
$$

is fulfilled.

For $\omega>0, i \omega$ is a root of (10) if and only if

$$
\begin{gathered}
\left(-\omega^{3} i+i m_{1} \omega+m_{2}\right) e^{i \omega \tau}+m_{3} \omega^{2} i+m_{4}+i m_{5} \omega e^{-i \omega \tau} \\
+m_{6} e^{-2 i \omega \tau}=0 .
\end{gathered}
$$

Separating the real and imaginary parts, we get

$$
\begin{gathered}
m_{2} \cos \omega \tau-\left(m_{1} \omega-\omega^{3}\right) \sin \omega \tau+m_{4} \\
+m_{5} \omega \sin \omega \tau=-m_{6} \cos 2 \omega \tau, \\
m_{2} \sin \omega \tau+\left(m_{1} \omega-\omega^{3}\right) \cos \omega \tau+m_{3} \omega^{2} \\
+m_{5} \omega \cos \omega \tau=m_{6} \sin 2 \omega \tau .
\end{gathered}
$$

It follows from (14) that

$$
\begin{aligned}
& {\left[m_{2} \cos \omega \tau-\left(m_{1} \omega-\omega^{3}\right) \sin \omega \tau+m_{4}\right.} \\
& \left.\quad+m_{5} \omega \sin \omega \tau\right]^{2}+\left[m_{2} \sin \omega \tau+\left(m_{1} \omega-\omega^{3}\right) \cos \omega \tau\right. \\
& \left.\quad+m_{3} \omega^{2}+m_{5} \omega \cos \omega \tau\right]^{2}=m_{6}^{2} .
\end{aligned}
$$

According to $\sin \omega \tau= \pm \sqrt{1-\cos ^{2} \omega \tau}$, then (15) takes the form

$$
\begin{aligned}
& {\left[m_{2} \cos \omega \tau-\left(m_{1} \omega-\omega^{3}\right)\left( \pm \sqrt{1-\cos ^{2} \omega \tau}\right)+m_{4}\right.} \\
& \left.\quad+m_{5} \omega\left( \pm \sqrt{1-\cos ^{2} \omega \tau}\right)\right]^{2}+\left[m_{2}\left( \pm \sqrt{1-\cos ^{2} \omega \tau}\right)\right. \\
& \left.\quad+\left(m_{1} \omega-\omega^{3}\right) \cos \omega \tau+m_{3} \omega^{2}+m_{5} \omega \cos \omega \tau\right]^{2} \\
& \quad=m_{6}^{2} .
\end{aligned}
$$

It is easy to see that (16) is equivalent to

$$
\begin{aligned}
& q_{1} \cos ^{4} \omega \tau+q_{2} \cos ^{3} \omega \tau+q_{3} \cos ^{2} \omega \tau+q_{4} \cos \omega \tau+q_{5} \\
& \quad=0
\end{aligned}
$$

where

$$
\begin{aligned}
& q_{1}=k_{1}^{2}+k_{5}^{2}, \\
& q_{2}=2\left(k_{2} k_{4}+k_{3} k_{5}\right), \\
& q_{3}=k_{2}^{2}+k_{3}^{2}-k_{5}^{2}-2 k_{4}\left(m_{6}^{2}-k_{1}\right), \\
& q_{4}=2 k_{2}\left(k_{1}-m_{6}^{2}\right)-2 k_{3} k_{5}, \\
& q_{5}=\left(m_{6}^{2}-k_{1}\right)^{2}-k_{3}^{2},
\end{aligned}
$$

where

$$
\begin{aligned}
k_{1}= & m_{2}^{2}+\left(m_{1} \omega-\omega^{3}\right)^{2}+m_{5}^{2} \omega^{2}+m_{4}^{2}+m_{3}^{2} \omega^{4}, \\
k_{2}= & 2 m_{2} m_{4}+2 m_{2} m_{5} \omega+2 m_{3} m_{5} \omega^{3} \\
& +2 m_{3} \omega^{2}\left(m_{1} \omega-\omega^{3}\right), \\
k_{3}= & 2 m_{4} m_{5} \omega-2 m_{4}\left(m_{1} \omega-\omega^{3}\right)+2 m_{2} m_{3} \omega^{2}, \\
k_{4}= & 2 m_{5} \omega\left(m_{1} \omega-\omega^{3}\right), \\
k_{5}= & 2 m_{2} m_{5} \omega .
\end{aligned}
$$

Let $\cos \omega \tau=r$ and denote

$$
l(r)=r^{4}+\frac{q_{2}}{q_{1}} r^{3}+\frac{q_{3}}{q_{1}} r^{2}+\frac{q_{4}}{q_{1}} r+\frac{q_{5}}{q_{1}} .
$$


It is easy to obtain that $l^{\prime}(r)=4 r^{3}+\left(3 q_{2} / q_{1}\right) r^{2}+\left(2 q_{3} / q_{1}\right) r+$ $q_{4} / q_{1}$. Set

$$
4 r^{3}+\frac{3 q_{2}}{q_{1}} r^{2}+\frac{2 q_{3}}{q_{1}} r+\frac{q_{4}}{q_{1}}=0 .
$$

Let $y=r+q_{2} / 4 q_{1}$. Then (21) becomes

$$
y^{3}+\gamma_{1} y+\gamma_{2}=0
$$

where $\gamma_{1}=q_{3} / 2 q_{1}-3 q_{2}^{2} / 16 q_{1}^{2}$ and $\gamma_{2}=q_{2}^{3} / 32 q_{1}^{3}-q_{2} q_{3} / 8 q_{1}^{2}+$ $q_{4} / 4 q_{1}$.

Define $\beta_{1}=\left(\gamma_{2} / 2\right)^{2}+\left(\gamma_{1} / 3\right)^{3}, \beta_{2}=(-1+i \sqrt{3}) / 2$. By $(22)$, then we obtain

$$
\begin{aligned}
& y_{1}=\sqrt[3]{-\frac{\gamma_{2}}{2}+\sqrt{\beta_{1}}}+\sqrt[3]{-\frac{\gamma_{2}}{2}-\sqrt{\beta_{1}}}, \\
& y_{2}=\sqrt[3]{-\frac{\gamma_{2}}{2}+\sqrt{\beta_{1}} \beta_{2}}+\sqrt[3]{-\frac{\gamma_{2}}{2}-\sqrt{\beta_{1}} \beta_{2}^{2}}, \\
& y_{3}=\sqrt[3]{-\frac{\gamma_{2}}{2}+\sqrt{\beta_{1}} \beta_{2}^{2}}+\sqrt[3]{-\frac{\gamma_{2}}{2}-\sqrt{\beta_{1}} \beta_{2}} .
\end{aligned}
$$

By the discussion above, we can obtain the expression of $\cos \omega \tau$, say

$$
\cos \omega \tau=f_{1}(\omega)
$$

where $f_{1}(\omega)$ is a function with respect to $\omega$. Substitute (24) into (15); then we can easily get the expression of $\sin \omega \tau$, say

$$
\sin \omega \tau=f_{2}(\omega)
$$

where $f_{2}(\omega)$ is a function with respect to $\omega$. Thus we obtain

$$
f_{1}^{2}(\omega)+f_{2}^{2}(\omega)=1
$$

If all the coefficients of system (2) are given, it is easy to use computer to calculate the roots of $(26)$ (say $\omega$ ). Then from (24), we derive

$$
\tau_{k}=\frac{1}{\omega}\left[\arccos f_{1}(\omega)+2 k \pi\right] \quad(k=0,1,2, \ldots) .
$$

Let $\lambda(\tau)=\alpha(\tau)+i \omega(\tau)$ be a root of (10) near $\tau=\tau_{k}$, $\alpha\left(\tau_{k}\right)=0$, and $\omega\left(\tau_{k}\right)=\omega_{k}$. Due to functional differential equation theory, for every $\tau_{k}, k=0,1,2,3, \ldots$, there exists $\varepsilon>0$ such that $\lambda(\tau)$ is continuously differentiable in $\tau$ for $\left|\tau-\tau_{k}\right|<\varepsilon$. Substituting $\lambda(\tau)$ into the left hand side of (10) and taking derivative with respect to $\tau$, we have

$$
\begin{aligned}
& {\left[\frac{d \lambda}{d \tau}\right]^{-1}} \\
& =-\frac{\left(3 \lambda^{2}+m_{1}\right) e^{-\lambda \tau}+2 m_{3} \lambda+m_{5} e^{-\lambda \tau}}{\lambda\left(\lambda^{3}+m_{1} \lambda+m_{2}\right) e^{\lambda \tau}-m_{5} \lambda^{2} e^{-\lambda \tau}-2 m_{6} \lambda e^{-2 \lambda \tau}} \\
& \quad-\frac{\tau}{\lambda} .
\end{aligned}
$$

Then

$$
\begin{aligned}
& {\left[\frac{d(\operatorname{Re} \lambda(\tau))}{d \tau}\right]_{\tau=\tau_{k}^{(j)}}^{-1}} \\
& =-\operatorname{Re}\left\{\frac{\left(3 \lambda^{2}+m_{1}\right) e^{-\lambda \tau}+2 m_{3} \lambda+m_{5} e^{-\lambda \tau}}{\lambda\left(\lambda^{3}+m_{1} \lambda+m_{2}\right) e^{\lambda \tau}-m_{5} \lambda^{2} e^{-\lambda \tau}-2 m_{6} \lambda e^{-2 \lambda \tau}}\right\}_{\tau=\tau_{k}} \\
& =-\operatorname{Re}\left\{\frac{A_{3}+i A_{4}}{A_{1}+i A_{2}}\right\}=\frac{A_{1} A_{3}+A_{2} A_{4}}{A_{1}^{2}+A_{2}^{2}},
\end{aligned}
$$

where

$$
\begin{aligned}
A_{1}= & \left(\omega_{k}^{4}-m_{1} \omega_{k}^{2}+m_{5} \omega_{k}^{2}\right) \cos \omega_{k} \tau_{k}-m_{2} \omega_{k} \sin \omega_{k} \tau_{k} \\
& -2 m_{6} \omega_{k} \sin 2 \omega_{k} \tau_{k}, \\
A_{2}= & \left(\omega_{k}^{4}-m_{1} \omega_{k}^{2}-m_{5} \omega_{k}^{2}\right) \sin \omega_{k} \tau_{k}+m_{2} \omega_{k} \cos \omega_{k} \tau_{k} \\
& -2 m_{6} \omega_{k} \cos 2 \omega_{k} \tau_{k}, \\
A_{3}= & \left(m_{1}+m_{5}-3 \omega_{k}^{2}\right) \cos \omega_{k} \tau_{k}, \\
A_{4}= & \left(3 \omega_{k}^{2}-m_{1}-m_{5}\right) \sin \omega_{k} \tau_{k}+2 m_{3} \omega_{k} .
\end{aligned}
$$

In order to obtain the main results in this paper, it is necessary to make the following assumption:

$$
A_{1} A_{3}+A_{2} A_{4} \neq 0 \text {. }
$$

In view of Lemma 1, it is easy to obtain the following result on stability and bifurcation of system (2).

Theorem 2. Suppose that (H1)-(H3) hold; then

(i) for system (2), its positive equilibrium $E_{0}\left(x_{1}^{*}, x_{2}^{*}, x_{3}^{*}\right)$ is asymptotically stable for $\tau \in\left[0, \tau_{0}\right)$;

(ii) system (2) undergoes a Hopf bifurcation at the positive equilibrium $E_{0}\left(x_{1}^{*}, x_{2}^{*}, x_{3}^{*}\right)$ when $\tau=\tau_{k}(k=0,1$, $2,3, \ldots)$; that is, system (2) has a branch of periodic solutions bifurcating from the positive equilibrium $E_{0}\left(x_{1}^{*}, x_{2}^{*}, x_{3}^{*}\right)$ near $\tau=\tau_{k}$.

\section{Direction and Stability of the Hopf Bifurcation}

In this section, we shall analyze the direction, stability, and period of these periodic solutions bifurcating from the positive equilibrium $E_{0}\left(x_{1}^{*}, x_{2}^{*}, x_{3}^{*}\right)$ at these critical value of $\tau$, by using techniques from normal form and center manifold theory [24]. Throughout this section, we always assume that system (2) undergoes Hopf bifurcation at the positive equilibrium $E_{0}\left(x_{1}^{*}, x_{2}^{*}, x_{3}^{*}\right)$ for $\tau=\tau_{k}, k=0,1,2,3, \ldots$, and then $\pm i \omega_{k}$ are corresponding purely imaginary roots of the characteristic equation at the positive equilibrium $E_{0}\left(x_{1}^{*}, x_{2}^{*}, x_{3}^{*}\right)$.

For convenience, let $\bar{x}_{i}(t)=x_{i}(\tau t)(i=1,2,3)$ and $\tau=$ $\tau_{k}+\mu$, where $\tau_{k}$ is defined by (27) and $\mu \in R$, drop the bar for the simplification of notations, and then system (4) can be written as an FDE in $\left.C=C([-1,0]), R^{3}\right)$ as

$$
\dot{u}(t)=L_{\mu}\left(u_{t}\right)+F\left(\mu, u_{t}\right),
$$


where $u(t)=\left(x_{1}(t), x_{2}(t), x_{3}(t)\right)^{T} \in C$ and $u_{t}(\theta)=u(t+\theta)=$ $\left(x_{1}(t+\theta), x_{2}(t+\theta), x_{3}(t+\theta)\right)^{T} \in C$, and $L_{\mu}: C \rightarrow R$ and $F: R \times C \rightarrow R$ are given by

$$
\begin{aligned}
L_{\mu} \phi= & \left(\tau_{k}+\mu\right)\left(\begin{array}{ccc}
0 & b_{2} & b_{3} \\
c_{1} & 0 & c_{3} \\
d_{1} & d_{2} & 0
\end{array}\right)\left(\begin{array}{l}
\phi_{1}(0) \\
\phi_{2}(0) \\
\phi_{3}(0)
\end{array}\right) \\
& +\left(\tau_{k}^{(j)}+\mu\right)\left(\begin{array}{lll}
b_{1} & 0 & 0 \\
0 & c_{2} & 0 \\
0 & 0 & d_{3}
\end{array}\right)\left(\begin{array}{l}
\phi_{1}(-1) \\
\phi_{2}(-1) \\
\phi_{3}(-1)
\end{array}\right), \\
f(\mu, \phi)= & \left(\tau_{k}+\mu\right)\left(\begin{array}{c}
f_{1}(\mu, \phi) \\
f_{2}(\mu, \phi) \\
f_{3}(\mu, \phi)
\end{array}\right),
\end{aligned}
$$

respectively, where $\phi(\theta)=\left(\phi_{1}(\theta), \phi_{2}(\theta), \phi_{3}(\theta)\right)^{T} \in C$ and

$$
\begin{aligned}
f_{1}(\mu, \phi)= & b_{4} \phi_{1}(0) \phi_{1}(-1)+b_{5} \phi_{1}(0) \phi_{2}(0) \\
& +b_{6} \phi_{1}(0) \phi_{3}(0), \\
f_{2}(\mu, \phi)= & c_{4} \phi_{1}(0) \phi_{2}(0)+c_{5} \phi_{2}(0) \phi_{2}(-1) \\
& +c_{6} \phi_{2}(0) \phi_{3}(0), \\
f_{3}(\mu, \phi)= & d_{4} \phi_{1}(0) \phi_{3}(0)+d_{5} \phi_{2}(0) \phi_{3}(0) \\
& +d_{6} \phi_{3}(0) \phi_{3}(-1) .
\end{aligned}
$$

From the discussion in Section 2, we know that if $\mu=0$, then system (31) undergoes a Hopf bifurcation at the positive equilibrium $E_{0}\left(x_{1}^{*}, x_{2}^{*}, x_{3}^{*}\right)$ and the associated characteristic equation of system (31) has a pair of simple imaginary roots $\pm \omega_{k} \tau_{k}$.

By the representation theorem, there is a matrix function with bounded variation components $\eta(\theta, \mu), \theta \in[-1,0]$, such that

$$
L_{\mu} \phi=\int_{-1}^{0} d \eta(\theta, \mu) \phi(\theta), \quad \text { for } \phi \in C
$$

In fact, we can choose

$$
\begin{aligned}
\eta(\theta, \mu)= & \left(\tau_{k}+\mu\right)\left(\begin{array}{ccc}
0 & b_{2} & b_{3} \\
c_{1} & 0 & c_{3} \\
d_{1} & d_{2} & 0
\end{array}\right) \delta(\theta) \\
& -\left(\tau_{k}+\mu\right)\left(\begin{array}{ccc}
b_{1} & 0 & 0 \\
0 & c_{2} & 0 \\
0 & 0 & d_{3}
\end{array}\right) \delta(\theta+1),
\end{aligned}
$$

where $\delta$ is the Dirac delta function.
For $\phi \in C\left([-1,0], R^{3}\right)$, define

$$
\begin{aligned}
A(\mu) \phi & = \begin{cases}\frac{d \phi(\theta)}{d \theta}, & -1 \leq \theta<0, \\
\int_{-1}^{0} d \eta(s, \mu) \phi(s), & \theta=0,\end{cases} \\
R \phi & = \begin{cases}0, & -1 \leq \theta<0, \\
f(\mu, \phi), & \theta=0 .\end{cases}
\end{aligned}
$$

Then (31) is equivalent to the abstract differential equation

$$
\dot{u}_{t}=A(\mu) u_{t}+R(\mu) u_{t},
$$

where $u_{t}(\theta)=u(t+\theta), \theta \in[-1,0]$.

For $\psi \in C\left([0,1],\left(R^{3}\right)^{*}\right)$, define

$$
A^{*} \psi(s)= \begin{cases}-\frac{d \psi(s)}{d s}, & s \in(0,1], \\ \int_{-1}^{0} d \eta^{T}(t, 0) \psi(-t), & s=0 .\end{cases}
$$

For $\phi \in C\left([-1,0], R^{3}\right)$ and $\psi \in C\left([0,1],\left(R^{3}\right)^{*}\right)$, define the bilinear form

$$
\begin{aligned}
\langle\psi, \phi\rangle= & \bar{\psi}(0) \phi(0) \\
& -\int_{-1}^{0} \int_{\xi=0}^{\theta} \psi^{T}(\xi-\theta) d \eta(\theta) \phi(\xi) d \xi,
\end{aligned}
$$

where $\eta(\theta)=\eta(\theta, 0), A=A(0)$, and $A^{*}$ are adjoint operators. By the discussions in Section 2, we know that $\pm i \omega_{k} \tau_{k}$ are eigenvalues of $A(0)$, and they are also eigenvalues of $A^{*}$.

Suppose that $q(\theta)=(1, \alpha, \beta)^{T} e^{i \omega_{k} \tau_{k} \theta}$ is the eigenvector of $A(0)$ corresponding to $i \omega_{k} \tau_{k}$; then $A(0) q(\theta)=i \omega_{k} \tau_{k} q(\theta)$. It follows from definition $A(0)$ and (32) and (35) that

$$
\begin{aligned}
& \tau_{k}\left(\begin{array}{ccc}
i \omega_{k}-b_{1} e^{-i \omega_{k} \tau_{k}} & -b_{2} & -b_{3} \\
-c_{1} & i \omega_{k}-c_{2} e^{-i \omega_{k} \tau_{k}} & -c_{3} \\
-d_{1} & -d_{2} & i \omega_{k}-d_{3} e^{-i \omega_{k} \tau_{k}}
\end{array}\right)\left(\begin{array}{l}
1 \\
\alpha \\
\beta
\end{array}\right) \\
& =\left(\begin{array}{l}
0 \\
0 \\
0
\end{array}\right) .
\end{aligned}
$$

Then we can obtain

$$
\begin{aligned}
& \alpha=\frac{c_{3}\left(b_{1} e^{-i \omega_{k} \tau_{k}}-i \omega_{k}\right)+b_{3} c_{1}}{b_{1} c_{3}-b_{3}\left(i \omega_{k}-c_{2} e^{-i \omega_{k} \tau_{k}}\right)}, \\
& \beta=\frac{\left(b_{1} e^{-i \omega_{k} \tau_{k}}-i \omega_{k}\right)\left(i \omega_{k}-c_{2} e^{-i \omega_{k} \tau_{k}}\right)}{b_{1} c_{3}-b_{3}\left(i \omega_{k}-c_{2} e^{-i \omega_{k} \tau_{k}}\right)} .
\end{aligned}
$$

Similarly, let $q^{*}(s)=D\left(1, \alpha^{*}, \beta^{*}\right) e^{i \omega_{k} \tau_{k} s}$ be the eigenvector of $A^{*}$ corresponding to $-i \omega_{k} \tau_{k}^{(j)}$; then $A^{*} q^{*}(\theta)=-i \omega_{k} \tau_{k} q^{*}(\theta)$. It follows from the definition $A^{*}$ that 


$$
D \tau_{k}\left(\begin{array}{ccc}
i \omega_{k}+b_{1} e^{-i \omega_{k} \tau_{k}} & c_{1} & d_{1} \\
b_{2} & i \omega_{k}+c_{2} e^{-i \omega_{k} \tau_{k}} & d_{2} \\
b_{3} & c_{3} & i \omega_{k}+d_{3} e^{-i \omega_{k} \tau_{k}}
\end{array}\right)\left(\begin{array}{c}
1 \\
\alpha^{*} \\
\beta^{*}
\end{array}\right)=\left(\begin{array}{l}
0 \\
0 \\
0
\end{array}\right)
$$

Hence

$$
\begin{aligned}
& \alpha^{*}=\frac{b_{2} c_{1}-d_{2}\left(i \omega_{k}+b_{1} e^{-i \omega_{k} \tau_{k}}\right)}{c_{1} d_{2}-d_{1}\left(i \omega_{k}+c_{2} e^{-i \omega_{k} \tau_{k}}\right)}, \\
& \beta^{*}=-\frac{\left(i \omega_{k}+c_{2} e^{-i \omega_{k} \tau_{k}}\right)\left(i \omega_{k}+b_{1} e^{-i \omega_{k} \tau_{k}}\right)-b_{2} c_{1}}{c_{1} d_{2}-d_{1}\left(i \omega_{k}+c_{2} e^{-i \omega_{k} \tau_{k}}\right)} .
\end{aligned}
$$

In order to assure $\left\langle q^{*}(s), q(\theta)\right\rangle=1$ and $\left\langle q^{*}(s), \bar{q}(\theta)\right\rangle=0$, we need to determine the value of $D$. From (40), we have

$$
\begin{aligned}
& \left\langle q^{*}(s), q(\theta)\right\rangle=\bar{D}\left(1, \overline{\alpha^{*}}, \overline{\beta^{*}}\right)(1, \alpha, \beta)^{T} \\
& -\int_{-1}^{0} \int_{\xi=0}^{\theta} \bar{D}\left(1, \overline{\alpha^{*}}, \overline{\beta^{*}}\right) e^{-i \omega_{k} \tau_{k}(\xi-\theta)} d \eta(\theta)(1, \alpha, \beta)^{T} \\
& \cdot e^{i \omega_{k} \tau_{k} \xi} d \xi=\bar{D}\left\{1+\alpha \overline{\alpha^{*}}+\beta \overline{\beta^{*}}-\int_{-1}^{0}\left(1, \overline{\alpha^{*}}, \overline{\beta^{*}}\right)\right. \\
& \left.\cdot \theta e^{i \omega_{k} \tau_{k} \theta} d \eta(\theta)(1, \alpha, \beta)^{T}\right\}=\bar{D}\left\{1+\alpha \overline{\alpha^{*}}+\beta \overline{\beta^{*}}\right. \\
& \left.+\tau_{k} e^{-i \omega_{k} \tau_{k}}\left(b_{1}+c_{2} \overline{\alpha^{*}} \alpha+d_{3} \overline{\beta^{*}} \alpha\right)\right\} .
\end{aligned}
$$

Thus we can choose

$$
D=\frac{1}{1+\bar{\alpha} \alpha^{*}+\bar{\beta} \beta^{*}+\tau_{k} e^{i \omega_{k} \tau_{k}}\left(b_{1}+c_{2} \alpha^{*} \bar{\alpha}+d_{3} \beta^{*} \bar{\beta}\right)} .
$$

Next, we use the same notations as those in Hassard et al. [24] and we first compute the coordinates to describe the center manifold $C_{0}$ at $\mu=0$. Let $u_{t}$ be the solution of (31) when $\mu=0$.

Define

$$
\begin{aligned}
z(t) & =\left\langle q^{*}, u_{t}\right\rangle, \\
W(t, \theta) & =u_{t}(\theta)-2 \operatorname{Re}\{z(t) q(\theta)\},
\end{aligned}
$$

on the center manifold $C_{0}$, and we have

$$
W(t, \theta)=W(z(t), \bar{z}(t), \theta)
$$

where

$$
\begin{aligned}
W(z(t), \bar{z}(t), \theta) & =W(z, \bar{z}) \\
& =W_{20} \frac{z^{2}}{2}+W_{11} z \bar{z}+W_{02} \frac{\bar{z}^{2}}{2}+\cdots,
\end{aligned}
$$

and $z$ and $\bar{z}$ are local coordinates for center manifold $C_{0}$ in the direction of $q^{*}$ and $\bar{q}^{*}$. Noting that $W$ is also real if $u_{t}$ is real, we consider only real solutions. For solutions $u_{t} \in C_{0}$ of (31)

$$
\begin{aligned}
\dot{z}(t)= & i \omega_{k} \tau_{k} z+\bar{q}^{*}(\theta) f(0, W(z, \bar{z}, \theta)) \\
& +2 \operatorname{Re}\{z q(\theta)\} \stackrel{\text { def }}{=} i \omega_{k} \tau_{k} z+\bar{q}^{*}(0) f_{0},
\end{aligned}
$$

which we write in abbreviated form as

$$
\dot{z}(t)=i \omega_{k} \tau_{k} z+g(z, \bar{z}),
$$

where

$$
g(z, \bar{z})=g_{20} \frac{z^{2}}{2}+g_{11} z \bar{z}+g_{02} \frac{\bar{z}^{2}}{2}+g_{21} \frac{z^{2} \bar{z}}{2}+\cdots .
$$

Hence we have

$$
\begin{aligned}
g(z, \bar{z})= & \bar{q}^{*}(0) f_{0}(z, \bar{z})=\bar{q}^{*}(0) f\left(0, u_{t}\right) \\
= & \tau_{k} \bar{D}\left(1, \overline{\alpha^{*}}, \overline{\beta^{*}}\right)\left(\begin{array}{c}
f_{1}\left(x_{1 t}, x_{2 t}, x_{3 t}\right) \\
f_{2}\left(x_{1 t}, x_{2 t}, x_{3 t}\right) \\
f_{3}\left(x_{1 t}, x_{2 t}, x_{3 t}\right)
\end{array}\right) \\
= & \bar{D} \tau_{k}\left(H_{1}+\overline{\alpha^{*}} H_{2}+\overline{\beta^{*}} H_{3}\right) z^{2} \\
& +2 \bar{D} \tau_{k}\left(P_{1}+\overline{\alpha^{*}} P_{2}+\overline{\beta^{*}} P_{3}\right) z \bar{z} \\
& +\bar{D} \tau_{k}\left(K_{1}+\overline{\alpha^{*}} K_{2}+\overline{\beta^{*}} K_{3}\right) \bar{z}^{2} \\
& +\bar{D} \tau_{k}\left(M_{1}+\overline{\alpha^{*}} M_{2}+\overline{\beta^{*}} M_{3}\right) z^{2} \bar{z}+\text { h.o.t., }
\end{aligned}
$$

where

$$
\begin{aligned}
& f_{1}\left(x_{1 t}, x_{2 t}, x_{3 t}\right)=b_{4} x_{1 t}(0) x_{1 t}(-1)+b_{5} x_{1 t}(0) x_{2 t}(0) \\
& +b_{6} x_{1 t}(0) x_{3 t}(0) \text {, } \\
& f_{2}\left(x_{1 t}, x_{2 t}, x_{3 t}\right)=c_{4} x_{1 t}(0) x_{2 t}(0)+c_{5} x_{2 t}(0) x_{2 t}(-1) \\
& +c_{6} x_{2 t}(0) x_{3 t}(0) \\
& f_{3}\left(x_{1 t}, x_{2 t}, x_{3 t}\right)=d_{4} x_{1 t}(0) x_{3 t}(0)+d_{5} x_{2 t}(0) x_{3 t}(0) \\
& +d_{6} x_{3 t}(0) x_{3 t}(-1) \text {, } \\
& H_{1}=b_{4} e^{-i \omega_{k} \tau_{k}}+b_{5} \alpha+b_{6} \beta \text {, } \\
& H_{2}=c_{4} \alpha+c_{5} \alpha e^{-i \omega_{k} \tau_{k}}+c_{6} \alpha \beta \text {, } \\
& H_{3}=d_{4} \beta+d_{5} \alpha \beta+d_{6} \beta^{2} e^{-i \omega_{k} \tau_{k}}, \\
& P_{1}=b_{4} \operatorname{Re}\left\{e^{i \omega_{k} \tau_{k}}\right\}+b_{5} \operatorname{Re}\{\alpha\}+b_{6} \operatorname{Re}\{\beta\} \text {, } \\
& P_{2}=c_{4} \operatorname{Re}\{\alpha\}+c_{5}|\alpha|^{2} \operatorname{Re}\{\alpha\}+c_{6} \operatorname{Re}\{\alpha \bar{\beta}\} \text {, }
\end{aligned}
$$




$$
\begin{aligned}
& P_{3}=d_{4} \operatorname{Re}\left\{\beta e^{i \omega_{k} \tau_{k}}\right\}+d_{5} \operatorname{Re}\{\alpha \bar{\beta}\}+d_{6}|\beta|^{2} \operatorname{Re}\left\{e^{i \omega_{k} \tau_{k}}\right\}, \\
& K_{1}=b_{4} \operatorname{Re}\left\{e^{i \omega_{k} \tau_{k}}\right\}+b_{5}\{\bar{\alpha}\}+b_{6}\{\bar{\beta}\} \text {, } \\
& K_{2}=c_{4} \bar{\alpha}+c_{5} \bar{\alpha}^{2} e^{i \omega_{k} \tau_{k}}+c_{6} \operatorname{Re}\{\bar{\alpha} \bar{\beta}\} \text {, } \\
& K_{3}=d_{4} \bar{\beta}+d_{5}\{\bar{\alpha} \bar{\beta}\}+d_{6} \bar{\beta}^{2} e^{i \omega_{k} \tau_{k}}, \\
& M_{1}=b_{4}\left[W_{11}^{(1)}(-1)+\frac{1}{2} W_{20}^{(1)}(-1)+W_{11}^{(1)}(0) e^{-i \omega_{k} \tau_{k}}\right. \\
& \left.+\frac{1}{2} W_{20}^{(1)}(0) e^{i \omega_{k} \tau_{k}}\right]+b_{5}\left[\frac{1}{2} W_{20}^{(2)}(0)+\frac{1}{2} W_{20}^{(1)}(0) \bar{\alpha}\right. \\
& \left.+W_{11}^{(2)}(0)+W_{11}^{(1)}(0)\right]+b_{6}\left[W_{11}^{(3)}(0)+\frac{1}{2} W_{20}^{(3)}(0)\right. \\
& \left.+\frac{1}{2} \bar{\beta} W_{20}^{(1)}(0)+W_{11}^{(1)}(0) \beta\right] \text {, } \\
& M_{2}=c_{4}\left[W_{11}^{(2)}(0)+\frac{1}{2} W_{20}^{(2)}(0)+\frac{1}{2} \bar{\alpha} W_{20}^{(1)}(0)\right. \\
& \left.+W_{11}^{(1)}(0)\right]+c_{5}\left[\alpha W_{11}^{(2)}(-1)+\frac{1}{2} \bar{\alpha} W_{20}^{(2)}(-1)\right. \\
& \left.+\alpha e^{-i \omega_{k} \tau_{k}} W_{11}^{(2)}(0)+\frac{1}{2} \bar{\alpha} W_{20}^{(2)}(0) e^{i \omega_{k} \tau_{k}}\right] \\
& +c_{6}\left[\alpha W_{11}^{(3)}(0)+\frac{1}{2} \bar{\alpha} W_{20}^{(3)}(0)+\frac{1}{2} \bar{\beta} W_{20}^{(2)}(0)\right. \\
& \left.+\beta W_{11}^{(2)}(0)\right], \\
& M_{3}=d_{4}\left[W_{11}^{(3)}(0)+\frac{1}{2} W_{20}^{(3)}(0)+\frac{1}{2} \bar{\beta} W_{20}^{(1)}(0)\right. \\
& \left.+\beta W_{20}^{(1)}(0)\right]+d_{5}\left[W_{11}^{(3)}(0) \alpha+\frac{1}{2} W_{20}^{(3)}(0) \bar{\alpha}\right. \\
& \left.+\frac{1}{2} W_{20}^{(2)}(0) \bar{\beta}+\beta W_{11}^{(2)}(0)\right]+d_{6}\left[W_{11}^{(3)}(-1) \beta\right. \\
& +\frac{1}{2} W_{20}^{(3)}(-1) \bar{\beta}+\beta e^{-i \omega_{k} \tau_{k}} W_{11}^{(3)}(0) \\
& \left.+\bar{\alpha} e^{i \omega_{k} \tau_{k}} W_{11}^{(3)}(0)\right] \text {. }
\end{aligned}
$$

Thus we obtain

$$
\begin{aligned}
& g_{20}=2 \bar{D} \tau_{k}\left(H_{1}+\overline{\alpha^{*}} H_{2}+\overline{\beta^{*}} H_{3}\right), \\
& g_{11}=2 \bar{D} \tau_{k}\left(P_{1}+\overline{\alpha^{*}} P_{2}+\overline{\beta^{*}} P_{3}\right), \\
& g_{02}=2 \bar{D} \tau_{k}\left(K_{1}+\overline{\alpha^{*}} K_{2}+\overline{\beta^{*}} K_{3}\right), \\
& g_{21}=2 \bar{D} \tau_{k} k\left(M_{1}+\overline{\alpha^{*}} M_{2}+\overline{\beta^{*}} M_{3}\right) .
\end{aligned}
$$

For unknown $W_{20}^{(i)}(0), W_{11}^{(i)}(0), W_{20}^{(i)}(-1), W_{11}^{(i)}(-1),(i=1$, $2,3)$ in $g_{21}$, we still need to compute them.
In view of (38) and (47), we have

$$
\begin{gathered}
\dot{W}= \begin{cases}A W-2 \operatorname{Re}\left\{\bar{q}^{*}(0) \bar{f} q(\theta)\right\}, & -1 \leq \theta<0, \\
A W-2 \operatorname{Re}\left\{\bar{q}^{*}(0) \bar{f} q(\theta)\right\}+\bar{f}, & \theta=0\end{cases} \\
\stackrel{\text { def }}{=} A W+H(z, \bar{z}, \theta),
\end{gathered}
$$

where

$$
H(z, \bar{z}, \theta)=H_{20}(\theta) \frac{z^{2}}{2}+H_{11}(\theta) z \bar{z}+H_{02}(\theta) \frac{\bar{z}^{2}}{2}
$$

Comparing the coefficients, we obtain

$$
\begin{aligned}
\left(A-2 i \tau_{k} \omega_{k}\right) W_{20} & =-H_{20}(\theta), \\
A W_{11}(\theta) & =-H_{11}(\theta) .
\end{aligned}
$$

We know that for $\theta \in[-1,0)$

$$
\begin{aligned}
H(z, \bar{z}, \theta) & =-\bar{q}^{*}(0) f_{0} q(\theta)-q^{*}(0) \bar{f}_{0} \bar{q}(\theta) \\
& =-g(z, \bar{z}) q(\theta)-\bar{g}(z, \bar{z}) \bar{q}(\theta) .
\end{aligned}
$$

Comparing the coefficients of (60) with (57) gives that

$$
\begin{aligned}
& H_{20}(\theta)=-g_{20} q(\theta)-\bar{g}_{02} \bar{q}(\theta), \\
& H_{11}(\theta)=-g_{11} q(\theta)-\bar{g}_{11} \bar{q}(\theta) .
\end{aligned}
$$

From (58), (61), and the definition of $A$, we get

$$
\dot{W}_{20}(\theta)=2 i \omega_{k} \tau_{k} W_{20}(\theta)+g_{20} q(\theta)+\overline{g_{02}} \bar{q}(\theta) .
$$

Noting that $q(\theta)=q(0) e^{i \omega_{k} \tau_{k} \theta}$, we have

$$
\begin{aligned}
W_{20}(\theta)= & \frac{i g_{20}}{\omega_{k} \tau_{k}} q(0) e^{i \omega_{k} \tau_{k} \theta}+\frac{i \bar{g}_{02}}{3 \omega_{k} \tau_{k}} \bar{q}(0) e^{-i \omega_{k} \tau_{k} \theta} \\
& +E_{1} e^{2 i \omega_{k} \tau_{k} \theta},
\end{aligned}
$$

where $E_{1}=\left(E_{1}^{(1)}, E_{1}^{(2)}, E_{1}^{(3)}\right)^{T} \in R^{3}$ is a constant vector.

Similarly, from (59), (62), and the definition of $A$, we have

$$
\begin{aligned}
\dot{W}_{11}(\theta)= & g_{11} q(\theta)+\overline{g_{11}} \bar{q}(\theta) \\
W_{11}(\theta)= & -\frac{i g_{11}}{\omega_{k} \tau_{k}} q(0) e^{i \omega_{k} \tau_{k} \theta}+\frac{i \bar{g}_{11}}{\omega_{k} \tau_{k}} \bar{q}(0) e^{-i \omega_{k} \tau_{k} \theta} \\
& +E_{2}
\end{aligned}
$$

where $E_{2}=\left(E_{2}^{(1)}, E_{2}^{(2)}, E_{2}^{(3)}\right)^{T} \in R^{3}$ is a constant vector.

In what follows, we shall seek appropriate $E_{1}$ and $E_{2}$ in (64) and (66), respectively. It follows from the definition of $A$, (61), and (62) that

$$
\int_{-1}^{0} d \eta(\theta) W_{20}(\theta)=2 i \omega_{k} \tau_{k} W_{20}(0)-H_{20}(0),
$$

$\int_{-1}^{0} d \eta(\theta) W_{11}(\theta)=-H_{11}(0)$, 
where $\eta(\theta)=\eta(0, \theta)$. From (58), we have

$$
\begin{aligned}
H_{20}(0)= & -g_{20} q(0)-\overline{g_{02}} \bar{q}(0)+2 \tau_{k}\left(H_{1}, H_{2}, H_{3}\right)^{T}, \\
H_{11}(0)= & -g_{11} q(0)-\overline{g_{11}}(0) \bar{q}(0) \\
& +2 \tau_{k}\left(P_{1}, P_{2}, P_{3}\right)^{T} .
\end{aligned}
$$

Noting that

$$
\begin{array}{r}
\left(i \omega_{k} \tau_{k} I-\int_{-1}^{0} e^{i \omega_{k} \tau_{k} \theta} d \eta(\theta)\right) q(0)=0, \\
\left(-i \omega_{k} \tau_{k} I-\int_{-1}^{0} e^{-i \omega_{k} \tau_{k} \theta} d \eta(\theta)\right) \bar{q}(0)=0,
\end{array}
$$

and substituting (64) and (69) into (67), we have

$$
\begin{aligned}
& \left(2 i \omega_{k} \tau_{k} I-\int_{-1}^{0} e^{2 i \omega_{k} \tau_{k} \theta} d \eta(\theta)\right) E_{1} \\
& =2 \tau_{k}^{(j)}\left(H_{1}, H_{2}, H_{3}\right)^{T} .
\end{aligned}
$$

That is,

$$
\begin{aligned}
& \left(\begin{array}{ccc}
2 i \omega_{k}-b_{1} e^{-2 i \omega_{k} \tau_{k}} & -b_{2} & -b_{3} \\
-c_{1} & 2 i \omega_{k}-c_{2} e^{-2 i \omega_{k} \tau_{k}^{(j)}} & -c_{3} \\
-d_{1} & -d_{2} & 2 i \omega_{k}-d_{3} e^{-2 i \omega_{k} \tau_{k}}
\end{array}\right) E_{1} \\
& =2\left(\begin{array}{l}
H_{1} \\
H_{2} \\
H_{3}
\end{array}\right) .
\end{aligned}
$$

It follows that

$$
\begin{aligned}
& E_{1}^{(1)}=\frac{\Delta_{11}}{\Delta_{1}}, \\
& E_{1}^{(2)}=\frac{\Delta_{12}}{\Delta_{1}}, \\
& E_{1}^{(3)}=\frac{\Delta_{13}}{\Delta_{1}},
\end{aligned}
$$

where

$$
\begin{aligned}
& \Delta_{1} \\
& =\operatorname{det}\left(\begin{array}{ccc}
2 i \omega_{k}-b_{1} e^{-2 i \omega_{k} \tau_{k}} & -b_{2} & -b_{3} \\
-c_{1} & 2 i \omega_{k}-c_{2} e^{-2 i \omega_{k} \tau_{k}} & -c_{3} \\
-d_{1} & -d_{2} & 2 i \omega_{k}-d_{3} e^{-2 i \omega_{k} \tau_{k}}
\end{array}\right) \text {, } \\
& \Delta_{11}=2 \operatorname{det}\left(\begin{array}{ccc}
H_{1} & -b_{2} & -b_{3} \\
H_{2} & 2 i \omega_{k}-c_{2} e^{-2 i \omega_{k} \tau_{k}} & -c_{3} \\
H_{3} & -d_{2} & 2 i \omega_{k}-d_{3} e^{-2 i \omega_{k} \tau_{k}}
\end{array}\right) \text {, } \\
& \Delta_{12}=2 \operatorname{det}\left(\begin{array}{ccc}
2 i \omega_{k}-b_{1} e^{-2 i \omega_{k} \tau_{k}} & H_{1} & -b_{3} \\
-c_{1} & H_{2} & -c_{3} \\
-d_{1} & H_{3} & 2 i \omega_{k}-d_{3} e^{-2 i \omega_{k} \tau_{k}}
\end{array}\right) \text {, } \\
& \Delta_{13}=2 \operatorname{det}\left(\begin{array}{ccc}
2 i \omega_{k}-b_{1} e^{-2 i \omega_{k} \tau_{k}} & -b_{2} & H_{1} \\
-c_{1} & 2 i \omega_{k}-c_{2} e^{-2 i \omega_{k} \tau_{k}} & H_{2} \\
-d_{1} & -d_{2} & H_{3}
\end{array}\right) \text {. }
\end{aligned}
$$

Similarly, substituting (65) and (70) into (68), we have

$$
\left(\int_{-1}^{0} d \eta(\theta)\right) E_{2}=2 \tau_{k}\left(P_{1}, P_{2}, P_{3}\right)^{T} .
$$

That is,

$$
\left(\begin{array}{lll}
b_{1} & b_{2} & b_{3} \\
c_{1} & c_{2} & c_{3} \\
d_{1} & d_{2} & d_{3}
\end{array}\right) E_{2}=2\left(\begin{array}{c}
-P_{1} \\
-P_{2} \\
-P_{3}
\end{array}\right)
$$

It follows that

$$
\begin{aligned}
& E_{2}^{(1)}=\frac{\Delta_{21}}{\Delta_{2}}, \\
& E_{2}^{(2)}=\frac{\Delta_{22}}{\Delta_{2}}, \\
& E_{2}^{(3)}=\frac{\Delta_{23}}{\Delta_{2}},
\end{aligned}
$$

where

$$
\begin{aligned}
& \Delta_{2}=\operatorname{det}\left(\begin{array}{lll}
b_{1} & b_{2} & b_{3} \\
c_{1} & c_{2} & c_{3} \\
d_{1} & d_{2} & d_{3}
\end{array}\right), \\
& \Delta_{21}=-\operatorname{det}\left(\begin{array}{lll}
P_{1} & b_{2} & b_{3} \\
P_{2} & c_{2} & c_{3} \\
P_{3} & d_{2} & d_{3}
\end{array}\right),
\end{aligned}
$$$$
\Delta_{22}=-\operatorname{det}\left(\begin{array}{lll}
b_{1} & P_{1} & b_{3} \\
c_{1} & P_{2} & c_{3} \\
d_{1} & P_{3} & d_{3}
\end{array}\right),
$$$$
\Delta_{23}=-\operatorname{det}\left(\begin{array}{lll}
b_{1} & b_{2} & P_{1} \\
c_{1} & c_{2} & P_{2} \\
d_{1} & d_{2} & P_{3}
\end{array}\right) .
$$

From (64), (66), (74), and (78), we can calculate $g_{21}$ and derive the following values:

$$
\begin{aligned}
c_{1}(0) & =\frac{i}{2 \omega_{k} \tau_{k}}\left(g_{20} g_{11}-2\left|g_{11}\right|^{2}-\frac{\left|g_{02}\right|^{2}}{3}\right)+\frac{g_{21}}{2}, \\
\mu_{2} & =-\frac{\operatorname{Re}\left\{c_{1}(0)\right\}}{\operatorname{Re}\left\{\dot{\lambda}\left(\tau_{k}\right)\right\}}, \\
\beta_{2} & =2 \operatorname{Re}\left(c_{1}(0)\right), \\
T_{2} & =-\frac{\operatorname{Im}\left\{c_{1}(0)\right\}+\mu_{2} \operatorname{Im}\left\{\lambda^{\prime}\left(\tau_{k}\right)\right\}}{\omega_{k} \tau_{k}} .
\end{aligned}
$$

These formulae give a description of the Hopf bifurcation periodic solutions of (31) at $\tau=\tau_{k},(k=0,1,2,3, \ldots)$ on the center manifold. From the discussion above, we have the following result. 


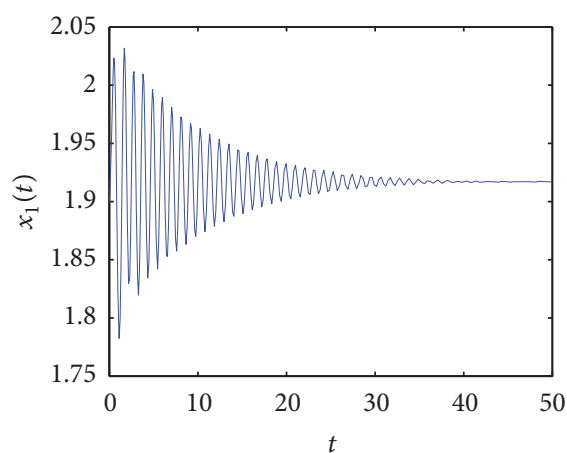

(a)

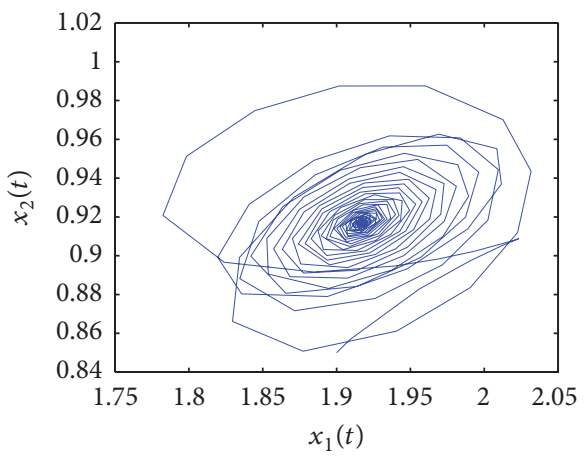

(d)

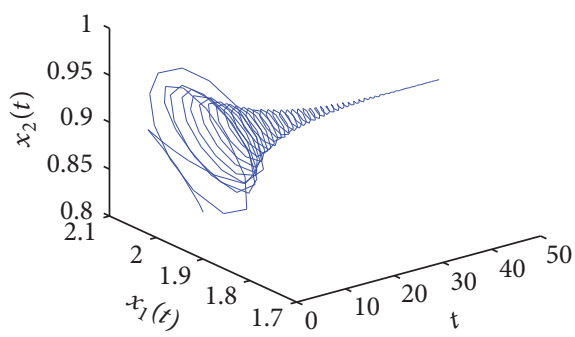

(g)

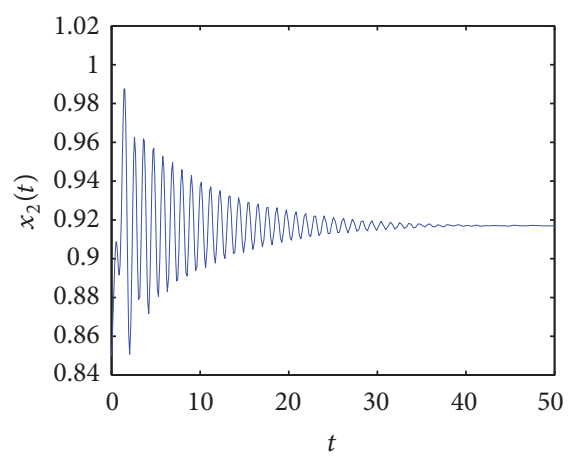

(b)

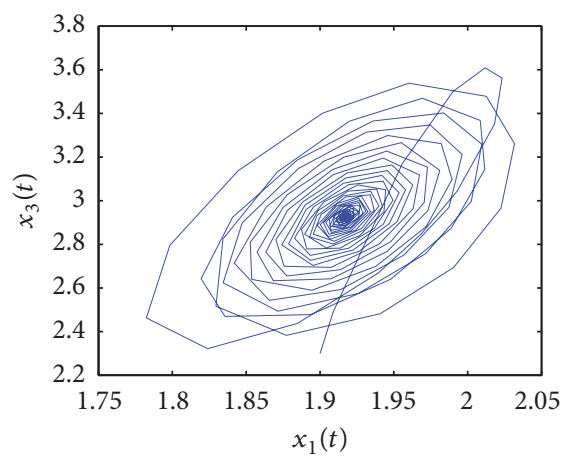

(e)

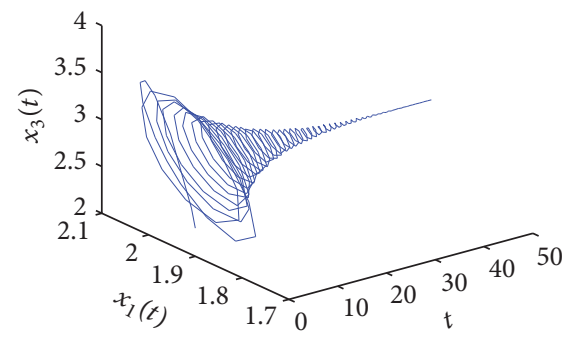

(h)

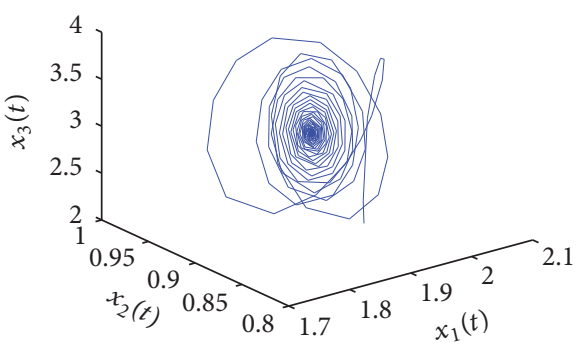

(j)

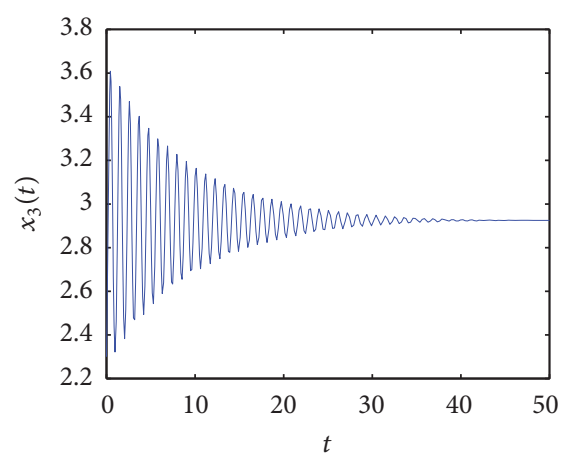

(c)

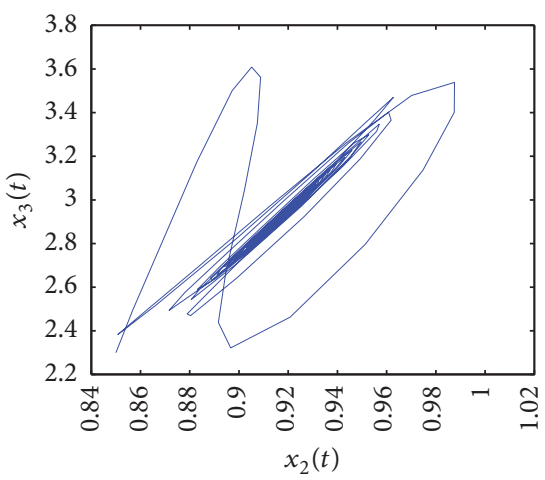

(f)

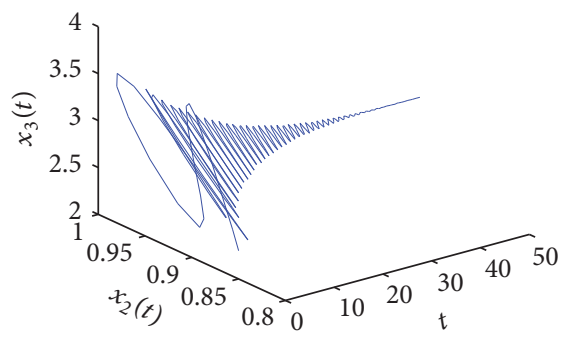

(i)

FIGURE 1: Dynamical behavior of system $(81)$ with $\tau=0.26<\tau_{0} \approx 0.265$. The positive equilibrium $E_{0}(1.9170,0.9170,2.9251)$ is asymptotically stable. The initial value $\left(x_{1}(0), x_{2}(0), x_{3}(0)\right)=(1.9,0.85,2.3)$.

Theorem 3. The direction of the Hopf bifurcation is forward (backward) if $\mu_{2}>0\left(\mu_{2}<0\right)$; the bifurcating periodic solutions are orbitally asymptotically stable with asymptotical phase (unstable) if $\beta_{2}<0\left(\beta_{2}>0\right)$; the periods of the bifurcating periodic solutions increase (decrease) if $T_{2}>$ $0\left(T_{2}<0\right)$.

\section{Numerical Examples}

In this section, numerical simulations are carried out to investigate the effect of the time delay on dynamical behaviors of system (2) as well as illustrate our theoretical results. For convenience, we assume that some parametric values of 


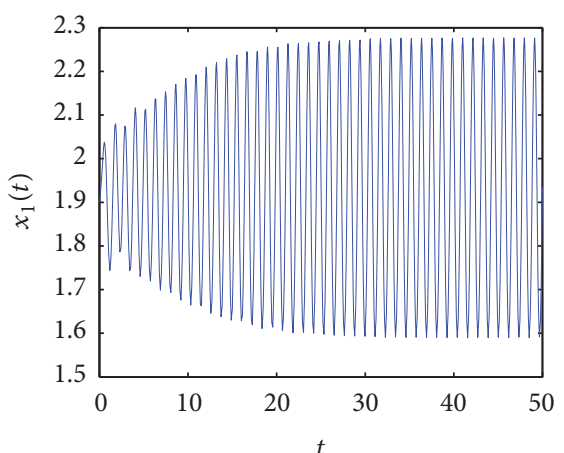

(a)

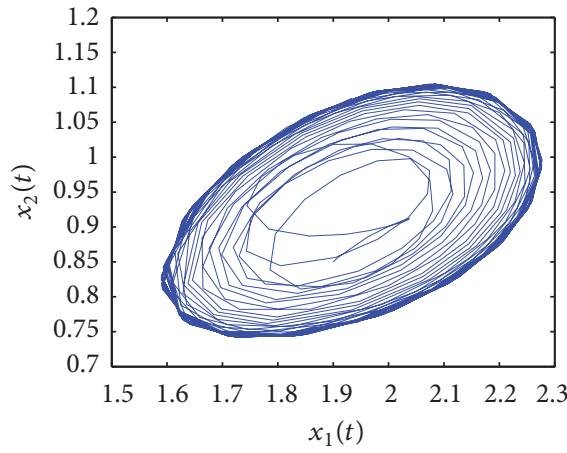

(d)

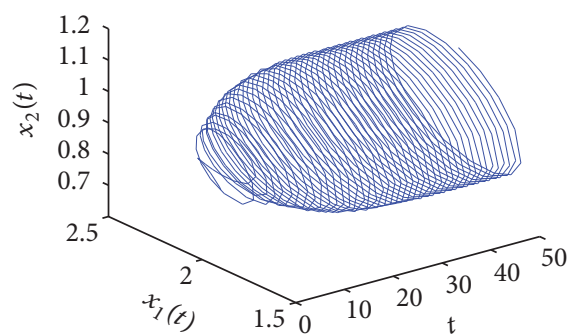

(g)

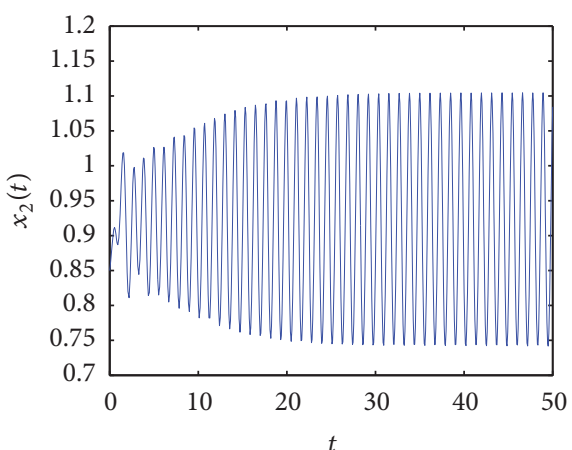

(b)

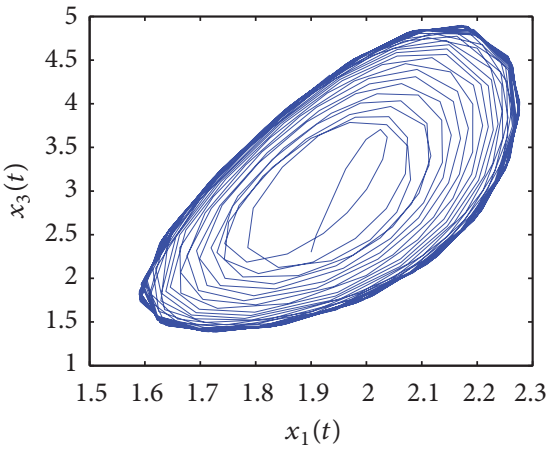

(e)

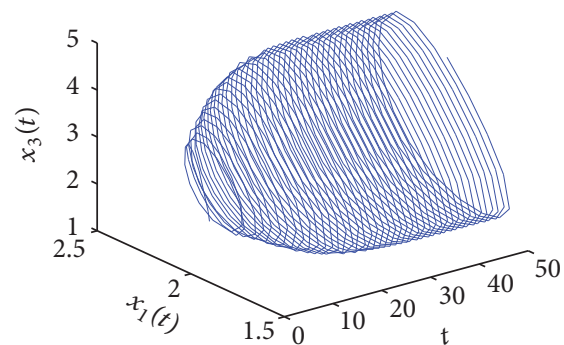

(h)

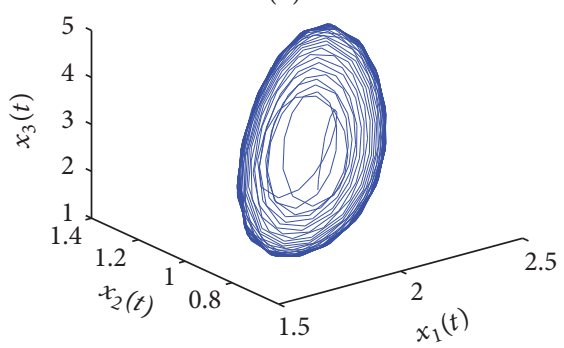

(j)

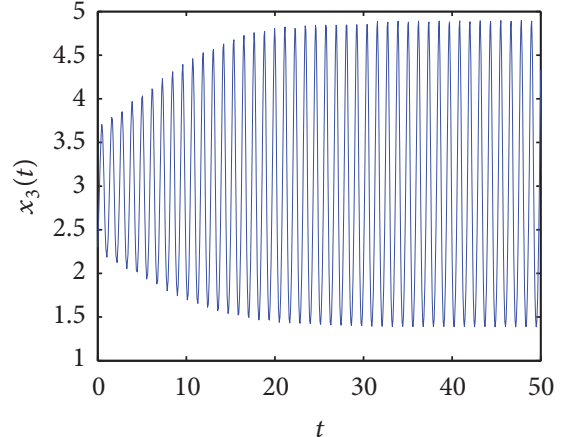

(c)

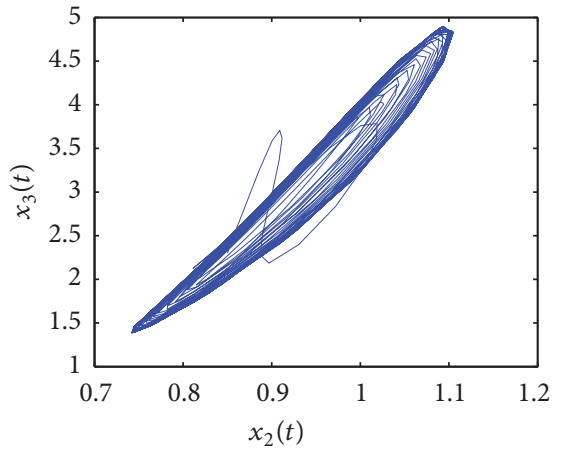

(f)

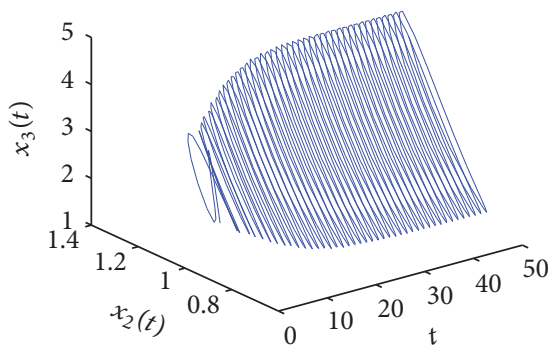

(i)

FIGURE 2: Dynamical behavior of system (81) with $\tau=0.28>\tau_{0} \approx 0.265$. Hopf bifurcation occurs from the positive equilibrium $E_{0}(1.9170,0.9170,2.9251)$. The initial value $\left(x_{1}(0), x_{2}(0), x_{3}(0)\right)=(1.9,0.85,2.3)$.

system (2) are kept as $r_{1}=7, r_{2}=6, r_{3}=5, a_{11}=3, a_{12}=$ 2, $a_{13}=0.2, a_{21}=2, a_{22}=3, a_{23}=0.2, a_{31}=0.3, a_{32}=$ 0.3 , and $a_{33}=2$. Then system (2) takes the form

$$
\begin{aligned}
& \dot{x}_{1}(t)=x_{1}(t)\left[7-3 x_{1}(t-\tau)-2 x_{2}(t)+0.2 x_{3}(t)\right], \\
& \dot{x}_{2}(t)=x_{2}(t)\left[6-2 x_{1}(t)-3 x_{2}(t-\tau)+0.2 x_{3}(t)\right], \\
& \dot{x}_{3}(t) \\
& \quad=x_{3}(t)\left[5+0.3 x_{1}(t)+0.3 x_{2}(t)-2 x_{3}(t-\tau)\right],
\end{aligned}
$$

which has a positive equilibrium $E_{0}(1.9170,0.9170,2.9251)$ and all the conditions given in Theorem 2 are satisfied. When $\tau=0$, the positive equilibrium $E_{0}(1.9170,0.9170,2.9251)$ is asymptotically stable. Fix $k=0$ and by means of Matlab 7.0 , we get $\omega_{0} \approx 0.9775, \tau_{0} \approx 0.265$, and $\dot{\lambda}\left(\tau_{0}\right) \approx 0.6505-$ 8.4322i. Thus we can derive the following values: $c_{1}(0) \approx$ $-2.3628-4.6636 i, \mu_{2} \approx 3.6323, \beta_{2} \approx-4.7256$, and $T_{2} \approx$ 136.2424. Furthermore, it follows that $\mu_{2}>0$ and $\beta_{2}<0$. 
Thus the positive equilibrium $E_{0}(1.9170,0.9170,2.9251)$ is stable when $\tau<\tau_{0}$. When $\tau=0.26<\tau_{0} \approx 0.265$, we can easily plot the time series of every population and phase portrait of the system and find that the solution of (81) with initial value $x_{1}(0)=1.9, x_{2}(0)=0.85$, and $x_{3}(0)=2.3$ would tend to a positive equilibrium $E_{0}(1.9170,0.9170,2.9251)$ (see Figures $1(\mathrm{a})-1(\mathrm{j})$, in detail); that is, the positive equilibrium $E_{0}(1.9170,0.9170,2.9251)$ is asymptotically stable. When $\tau$ passes through the critical value $\tau_{0} \approx 0.265$, the positive equilibrium $E_{0}(1.9170,0.9170,2.9251)$ loses its stability and a Hopf bifurcation occurs; that is, a family of periodic solutions bifurcate from the positive equilibrium $E_{0}(1.9170,0.9170,2.9251)$. Figures $2(\mathrm{a})-2(\mathrm{j})$ are plotted by fixing the time delay $\tau=1$ and initial value $x_{1}(0)=$ $1.9, x_{2}(0)=0.85$, and $x_{3}(0)=2.3$. It is shown that a Hopf bifurcation occurs from the positive equilibrium $E_{0}(1.9170$, $0.9170,2.9251)$. In view of $\mu_{2}>0$ and $\beta_{2}<0$, we find that the direction of the Hopf bifurcation is $\tau>\tau_{0} \approx 0.265$ and these bifurcating periodic solutions from $E_{0}(1.9170$, $0.9170,2.9251$ ) at $\tau_{0} \approx 0.265$ are stable.

\section{Biological Meanings and Conclusions}

In this paper, the dynamics including the local stability of the positive equilibrium $E_{0}\left(x_{1}^{*}, x_{2}^{*}, x_{3}^{*}\right)$ and the local Hopf bifurcation of a competitor-competitor-mutualist Lotka-Volterra model are investigated. It is showed that the positive equilibrium $E_{0}\left(x_{1}^{*}, x_{2}^{*}, x_{3}^{*}\right)$ of system (2) is asymptotically stable for all $\tau \in\left[0, \tau_{0}\right)$ when the conditions (H1)- $(\mathrm{H} 3)$ are satisfied. This reveals that the densities of competing species $x_{1}, x_{2}$ and cooperating species $x_{3}$ will tend to stabilization; in other words, the densities of competing species $x_{1}, x_{2}$ and cooperating species will tend to $x_{1}^{*}, x_{2}^{*}, x_{3}^{*}$, respectively, and this situation does not vary with the delay $\tau \in\left[0, \tau_{0}\right)$. When the delay $\tau$ increases, the positive equilibrium $E_{0}\left(x_{1}^{*}, x_{2}^{*}, x_{3}^{*}\right)$ loses its stability and a sequence of Hopf bifurcations occur from the positive equilibrium $E_{0}\left(x_{1}^{*}, x_{2}^{*}, x_{3}^{*}\right)$; that is, a family of periodic orbits bifurcate from the positive equilibrium $E_{0}\left(x_{1}^{*}, x_{2}^{*}, x_{3}^{*}\right)$. From this we can find that competing species $x_{1}, x_{2}$ and cooperating species $x_{3}$ can coexist and keep in periodic mode; that is, the densities of competing species $x_{1}, x_{2}$ and cooperating species $x_{3}$ will oscillate in the vicinity of $x_{1}^{*}, x_{2}^{*}$, and $x_{3}^{*}$, respectively. Finally, the direction of Hopf bifurcation and the stability of the bifurcating periodic orbits are discussed by applying the normal form theory and the center manifold theorem. A numerical example is also included to verify our theoretical findings.

\section{Conflicts of Interest}

The author declares that there are no conflicts of interest.

\section{Acknowledgments}

This work is supported by National Natural Science Foundation of China (no. 61673008 and no. 11261010) and Project of High-Level Innovative Talents of Guizhou Province ([2016]5651).

\section{References}

[1] G. Aggelis, D. V. Vayenas, V. Tsagou, and S. Pavlou, "Preypredator dynamics with predator switching regulated by a catabolic repression control mode," Ecological Modelling, vol. 183, no. 4, pp. 451-462, 2005.

[2] N. Bairagi and D. Jana, "On the stability and Hopf bifurcation of a delay-induced predator-prey system with habitat complexity," Applied Mathematical Modelling. Simulation and Computation for Engineering and Environmental Systems, vol. 35, no. 7, pp. 3255-3267, 2011.

[3] K. Chakraborty, M. Chakraborty, and T. K. Kar, "Bifurcation and control of a bioeconomic model of a prey-predator system with a time delay," Nonlinear Analysis: Hybrid Systems, vol. 5, no. 4, pp. 613-625, 2011.

[4] K. pada Das, K. Kundu, and J. Chattopadhyay, "A predatorprey mathematical model with both the populations affected by diseases," Ecological Complexity, vol. 8, no. 1, pp. 68-80, 2011.

[5] C.-H. Hsu, C.-R. Yang, T.-H. Yang, and T.-S. Yang, "Existence of traveling wave solutions for diffusive predator-prey type systems," Journal of Differential Equations, vol. 252, no. 4, pp. 3040-3075, 2012.

[6] J. Jiao, S. Cai, and L. Chen, "Analysis of a stage-structured predatory-prey system with birth pulse and impulsive harvesting at different moments," Nonlinear Analysis: Real World Applications, vol. 12, no. 4, pp. 2232-2244, 2011.

[7] T. K. Kar and A. Ghorai, "Dynamic behaviour of a delayed predator-prey model with harvesting," Applied Mathematics and Computation, vol. 217, no. 22, pp. 9085-9104, 2011.

[8] W. Ko and K. Ryu, "Coexistence states of a nonlinear LotkaVolterra type predator-prey model with cross-diffusion," Nonlinear Analysis: Theory, Methods \& Applications, vol. 71, no. 12, pp. 1109-1115, 2009.

[9] Q. M. Liu and R. Xu, "Periodic solution for a delayed threespecies food-chain system with Holling type-II functional response," International Journal of Mathematics and Mathematical Sciences, vol. 64, pp. 4057-4070, 2003.

[10] Y. Saito, J. Sugie, and Y.-H. Lee, "Global asymptotic stability for predator-prey models with environmental time-variations," Applied Mathematics Letters, vol. 24, no. 12, pp. 1973-1980, 2011.

[11] Y. Song and J. Wei, "Local Hopf bifurcation and global periodic solutions in a delayed predator-prey system," Journal of Mathematical Analysis and Applications, vol. 301, no. 1, pp. 1-21, 2005.

[12] X. Lv, P. Yan, and S. Lu, "Existence and global attractivity of positive periodic solutions of competitor-competitor-mutualist Lotka-Volterra systems with deviating arguments," Mathematical and Computer Modelling, vol. 51, no. 5-6, pp. 823-832, 2010.

[13] M. Xiao and J.-D. Cao, "Stability and Hopf bifurcation in a delayed competitive web sites model," Physics Letters A, vol. 353, no. 2-3, pp. 138-150, 2006.

[14] C. Xu, X. Tang, and M. Liao, "Stability and bifurcation analysis of a delayed predator-prey model of prey dispersal in two-patch environments," Applied Mathematics and Computation, vol. 216, no. 10, pp. 2920-2936, 2010.

[15] C. Xu, X. Tang, M. Liao, and X. He, "Bifurcation analysis in a delayed Lokta-Volterra predator-prey model with two delays," Nonlinear Dynamics, vol. 66, no. 1-2, pp. 169-183, 2011.

[16] R. Xu and Z. E. Ma, "Stability and Hopf bifurcation in a ratiodependent predator-prey system with stage structure," Chaos, Solitons \& Fractals, vol. 38, no. 3, pp. 669-684, 2008. 
[17] X.-P. Yan and W.-T. Li, "Bifurcation and global periodic solutions in a delayed facultative mutualism system," Physica D. Nonlinear Phenomena, vol. 227, no. 1, pp. 51-69, 2007.

[18] X.-P. Yan and C.-H. Zhang, "Hopf bifurcation in a delayed Lokta-Volterra predator-prey system," Nonlinear Analysis: Real World Applications, vol. 9, no. 1, pp. 114-127, 2008.

[19] C. B. Yu and J. J. Wei, "Stability and bifurcation analysis in a basic model of the immune response with delays," Chaos, Solitons \& Fractals, vol. 41, no. 3, pp. 1223-1234, 2009.

[20] S. Yuan and F. Zhang, "Stability and global Hopf bifurcation in a delayed predator-prey system," Nonlinear Analysis: Real World Applications, vol. 11, no. 2, pp. 959-977, 2010.

[21] M. L. Zeeman, "Hopf bifurcations in competitive threedimensional Lotka-Volterra systems," Dynamics and Stability of Systems, vol. 8, no. 3, pp. 189-217, 1993.

[22] X. Zhou, X. Shi, and X. Song, "Analysis of nonautonomous predator-prey model with nonlinear diffusion and time delay," Applied Mathematics and Computation, vol. 196, no. 1, pp. 129136, 2008.

[23] S. G. Ruan and J. J. Wei, "On the zeros of transcendental functions with applications to stability of delay differential equations with two delays," Dynamics of Continuous, Discrete \& Impulsive Systems A: Mathematical Analysis, vol. 10, no. 6, pp. 863-874, 2003.

[24] B. Hassard, D. Kazarino, and Y. Wan, Theory and Applications of Hopf Bifurcation, Cambridge University Press, Cambridge, UK, 1981. 


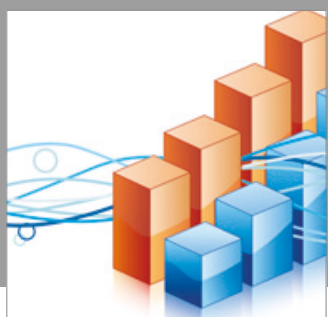

Advances in

Operations Research

vatersals

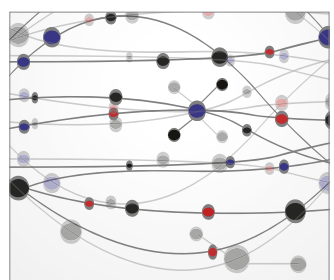

\section{The Scientific} World Journal
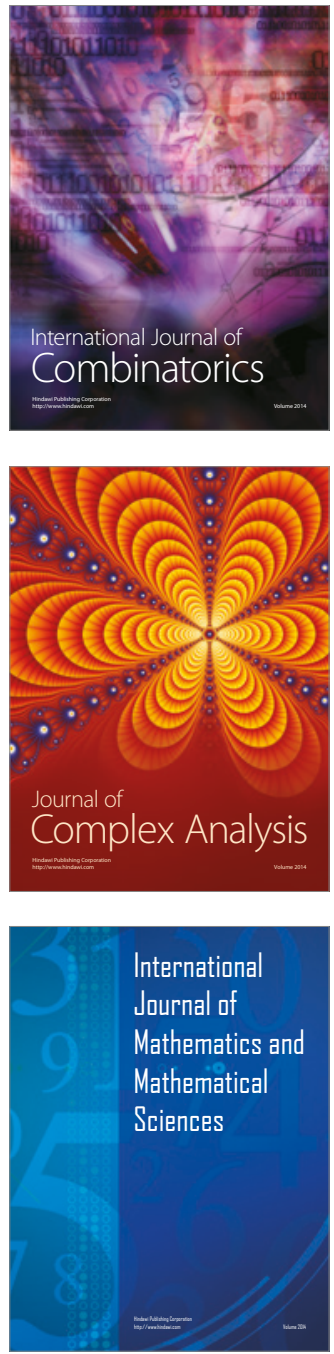
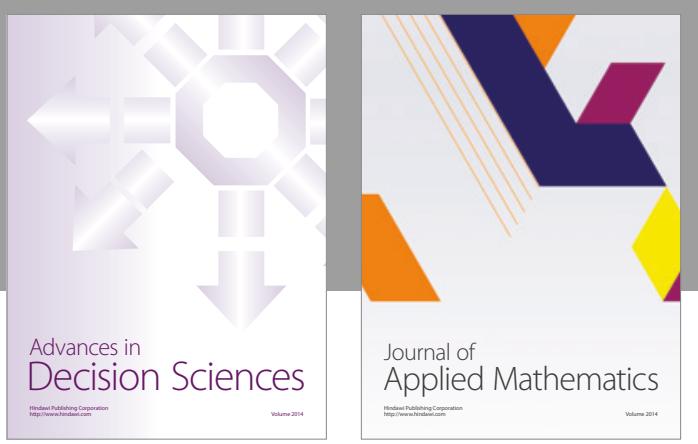

Algebra

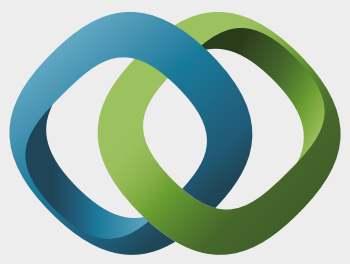

\section{Hindawi}

Submit your manuscripts at

https://www.hindawi.com
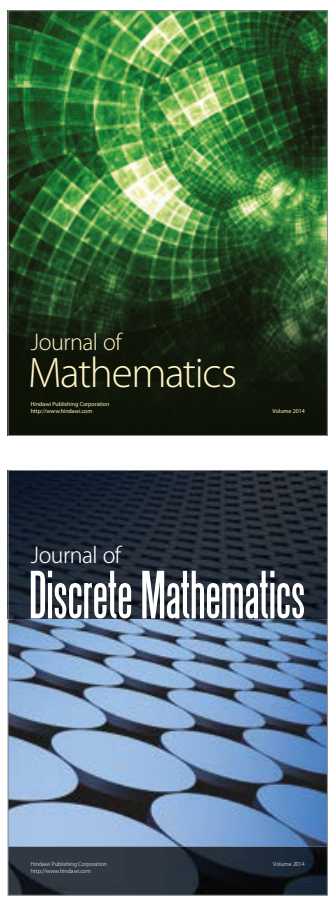

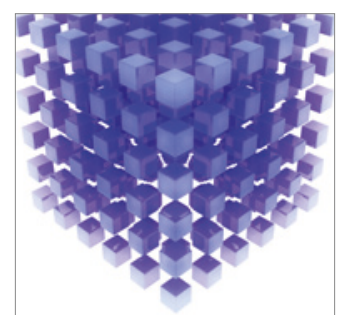

Mathematical Problems in Engineering
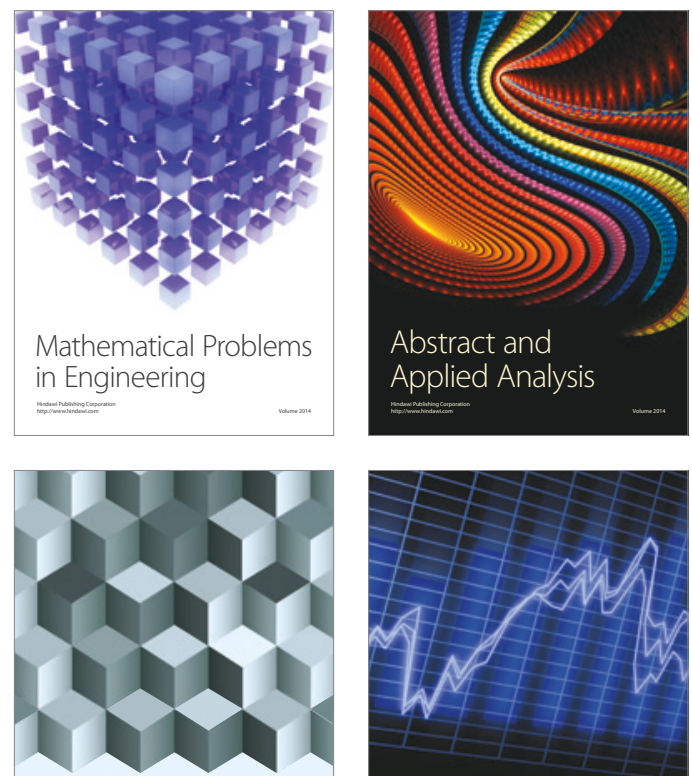

Journal of

Function Spaces

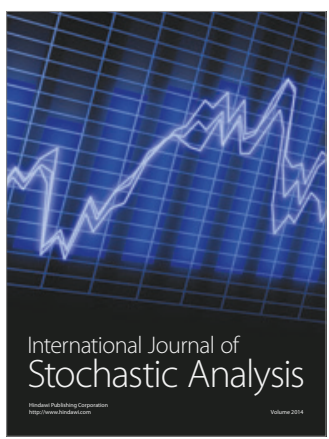

Probability and Statistics
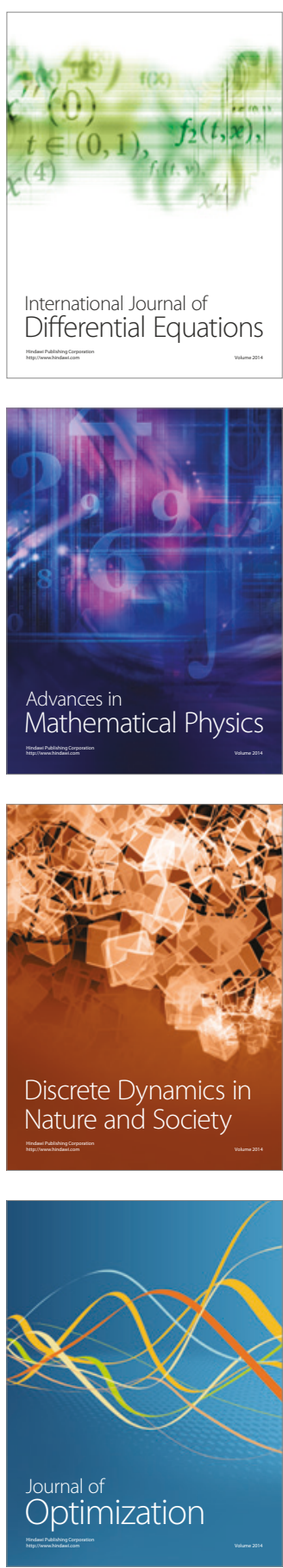